\title{
BRCA1 Is a Novel Prognostic Indicator and Associates with Immune Cell Infiltration in Hepatocellular Carcinoma
}

\author{
Jie Mei, Runjie Wang, Dandan Xia, Xuejing Yang, Weijian Zhou, Huiyu Wang, and Chaoying Liu
}

\begin{abstract}
The breast cancer gene 1 (BRCA1) is a tumor suppressor, and mutations or epigenetic inactivation will increase the risk of breast cancer oncogenesis. The current research aimed to explore the relationship between BRCA1 expression, prognosis, and tumor immunity in hepatocellular carcinoma (HCC). In this study, BRCA1 expression was analyzed via multiple online databases and its association with clinical characteristics, prognosis and genetic alterations was identified using the original The Cancer Genome Atlas-liver hepatocellular carcinoma cohorts. DNA methylation sites and their prognostic values were analyzed using MethSurv. The correlations between BRCA1 and immune infiltration were investigated via Tumor Immune Estimation Resource. As results, BRCA1 was significantly upregulated in tumor tissues in multiple HCC cohorts. Besides, high BRCA1 expression was correlated with race, advanced T stage, clinical stage, poor tumor grade, MSI status, and worse prognosis. Notably, BRCA1 expression was positively correlated with infiltration levels of B cells, CD4+ T cells, CD8+ T cells, neutrophils, macrophages, and dendritic cells. The current findings imply that BRCA1 is associated with prognosis and immune infiltration, laying foundations for in-depth research on the role of BRCA1 in HCC.
\end{abstract}

Keywords: BRCA1, bioinformatics, gene expression, prognosis, HCC

\section{Introduction}

$\mathbf{H}$ EPATOCELLULAR CARCINOMA (HCC) is one of the most widespread malignancies in the digestive system in the world, which may lead to thousands of tumor-related deaths. The American Cancer Society predicts that there will be about 42,810 new-onset new HCC cases and more than 30,000 deaths in the United States in 2020 (Siegel et al., 2020). About half of all HCC patients in the world are concentrated in China, which seriously endangers the health of people (Ding and Wang, 2014).

As we all know, symptoms of early HCC tend to be not obvious, leading to more than half of patients with primary HCC diagnosed with intermediate or advanced stage. Thus, the prognosis of HCC is pretty unsatisfactory. Besides, the depressing issue for $\mathrm{HCC}$ is that the 5-year overall survival (OS) rate is only 30-40\% (Tai et al., 2017). Although growing numbers of researches focusing on prognostic biomarkers have been carried out (De Stefano et al., 2018; $\mathrm{Gu}$ et al., 2020), it is still essential to in-depth investigate more potential reliable biomarkers to predict and monitor the prognosis of $\mathrm{HCC}$ patients.

The breast cancer gene 1 (BRCA1) encodes a nuclear phosphoprotein that functions as regulators in maintaining genomic stability, and it has been confirmed to be a tumor suppressor in breast cancer (Savage and Harkin, 2015). Downregulation of BRCA1 on account of inherited mutations or epigenetic inactivation predisposes to breast and ovarian cancer (Romagnolo et al., 2015). Several studies have confirmed that the expression of BRCA1 in breast and ovarian cancer is significantly downregulated and associated advanced stages (Shilpa et al., 2014; Hedau et al., 2015). Although increasing numbers of studies turn attention to the molecular functions and clinical associations of BRCA1 in multiple cancers, its exact prognostic roles have not been defined.

In the past decades, many visible and user-friendly online tools dependent on the The Cancer Genome Atlas (TCGA) dataset enormously enhanced the analysis efficiency of TCGA data (Zheng et al., 2020), and a mass of cancerrelated indicators have been identified based on the strength of these online platforms (Mei et al., 2019; Sun et al., 2019; Yuan et al., 2019). In this research, we applied these interactive tools to investigate the transcriptional expression and prognostic value of BRCA1 in multiple cancers. Besides, we further conducted an in-depth analysis of BRCA1 in HCC using original TCGA data. Consequently, our research systematically summarizes the expression of BRCA1 and discusses the potential prognostic value and immune infiltrating association of BRCA1 in HCC.

Department of Oncology, Wuxi People's Hospital Affiliated to Nanjing Medical University, Wuxi, Jiangsu, China. 


\section{Materials and Methods}

\section{HCCDB analysis}

HCCDB, the Integrative Molecular Database of Hepatocellular Carcinoma, is a gene expression atlas of HCC, containing 15 public HCC transcriptional expression datasets, totally 3917 samples (Lian et al., 2018). HCCDB offers the visualization for the findings from multiple bioinformatics analyses, such as differential expression analysis, prognostic analysis, and tissue-specific and tumor-specific expression analysis. Samples including BRCA1 expression data in HCCDB were used to analyze the expression of BRCA1 in tumor and normal tissues, to define the expression patterns of BRCA in HCC. This research is based on bioinformatics analysis, thus ethics approval is not applicable.

\section{Kaplan-Meier plotter database analysis}

Kaplan-Meier plotter is an online tool comprising gene expression data, clinical information, and survival data of multiple types of cancer patients (Lanczky et al., 2016). The pan-cancer analysis of BRCA1 prognostic values was analyzed using all cancer samples available on KaplanMeier plotter. The patients' cohorts were split at the median expression of BRCA1 transcriptional level, other parameters are default. All cohorts were visualized with Kaplan-Meier survival plots. Hazard ratio (HR), 95\% confidence interval (CI), and $\log$-rank $p$ value were calculated and displayed online.

\section{Human Protein Atlas database analysis}

Human Protein Atlas is launched with the aim to describe all the human proteins in cells, tissues, and organs using integration of multi-omics (Uhlen et al., 2010, 2015). All the data in the platform allow all researchers to freely acquire the data for definition of the human proteome. In this research, the level of BRCA1 protein expression in human liver tissues and HCC tissues were compared using data generated by Human Protein Atlas.

\section{MethSurv database analysis}

MethSurv is a user-friendly, comprehensive bioinformatics tool for visualization of methylation data and their association with prognosis (Modhukur et al., 2018). The DNA methylation sites of BRCA1 in TCGA were analyzed by MethSurv. Meanwhile, the prognostic values of all the methylation sites in BRCA1 gene were also investigated.

\section{Tumor Immune Estimation Resource database analysis}

Tumor Immune Estimation Resource (TIMER) database is an online tool for systematic analysis of immune cell infiltration across diverse cancer types from TCGA (Li et al., 2017). TIMER uses a deconvolution algorithm to estimate the abundance of tumor-infiltrating immune cells (TIICs) based on gene expression profiles (Li et al., 2016). We evaluated BRCA1 expression in multiple cancers and the correlation of BRCA1 expression with the abundance of immune cell infiltration, including B cells, CD8+ T cells, CD4+ T cells, neutrophils, macrophages, and dendritic cells (DCs), and the tumor purity. Besides, the correlation between
BRCA1 expression and markers of different subsets of immune cells was also analyzed by TIMER.

\section{TCGA-liver hepatocellular carcinoma dataset analysis}

The level 3 data of TCGA-liver hepatocellular carcinoma (TCGA-LIHC) and clinical data were obtained from the cBioPortal (Cerami et al., 2012; Gao et al., 2013). A total of 348 cases containing both mutations, copy-number alterations (CNA) from GISTIC, and BRCA1 mRNA expression RSEM, a data format of TCGA, were reserved to further analysis. Besides, paired clinical information of these cases was also downloaded.

Expression of BRCA1 in samples with different clinical characteristics and genetic alterations in HCC were analyzed using downloaded data. Based on the expression of BRCA1, total cases were ranked from low expression to high expression. The bottom $50 \%$ of patients were divided into the low expression group and the top $50 \%$ belonged to the high expression group. Next, Kaplan-Meier plots were plotted to evaluate the prognostic value of BRCA1. Furthermore, prognostic value of BRCA1 was analyzed by Kaplan-Meier curves and univariate and multivariate analyses. Moreover, the correlations between BRCA and DNA repair genes were analyzed by Pearson correlation test.

\section{Statistical analysis}

Most statistical analyses were performed on the bioinformatics database online. The differential mRNA expression of BRCA1 in HCC tissues was analyzed by Student's $t$-test or one-way analysis of variance (ANOVA). KaplanMeier survival plots were generated with survival curves compared by log-rank test. The threshold between high and low expression of BRCA1 was defined as the median level of BRCA1 expression in the whole research. For all analyses, differences were considered statistically significant if $p$ values were $<0.05$.

\section{Results}

Pan-cancer analysis of BRCA1 expression profiles and prognostic values

As depicted in Figure 1, BRCA1 expression was remarkably overexpressed in most types of solid cancers, including bladder urothelial carcinoma (BLCA), breast invasive carcinoma (BRCA), cholangiocarcinoma (CHOL), colon adenocarcinoma (COAD), esophageal carcinoma (ESCA), head and neck cancer (HNSC), kidney renal clear cell carcinoma (KIRC), kidney renal papillary carcinoma (KIRP), liver hepatocellular carcinoma (LIHC), lung adenocarcinoma (LUAD), lung squamous cell carcinoma (LUSC), rectum adenocarcinoma (READ), stomach adenocarcinoma (STAD), and uterine corpus endometrial carcinoma (UCEC), whereas only kidney chromophobe (KICH) tissues expressed lower BRCA1 compared with that in normal tissues (Fig. 1A).

Next, the prognostic values of BRCA1 in multiple cancers were analyzed by Kaplan-Meier plotter database. BRCA1 had controversial prognostic values in different cancers. Briefly, high expression of BRCA1 was correlated 

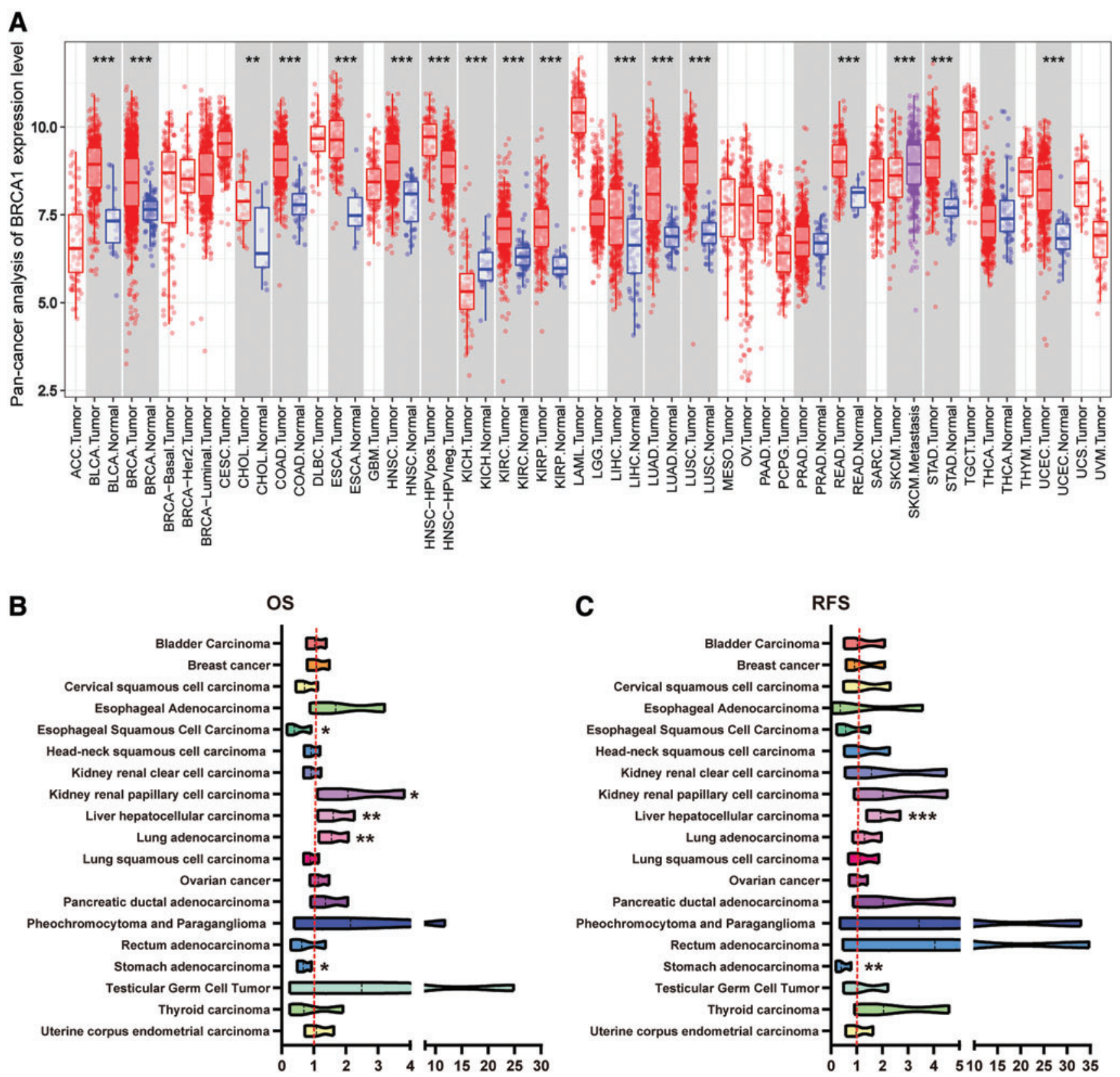

FIG. 1. Expression and prognostic values of BRCA1 in different types of human cancers. (A) The level of BRCA1 expression in different tumor types from the TCGA database in TIMER. (B) Forest plot displaying the prognostic values of BRCA1 for OS generated by using the Kaplan-Meier plotter database. (C) Forest plot displaying the prognostic values of BRCA1 for RFS generated by using the Kaplan-Meier plotter database. Note: $* p<0.05, * * p<0.01, * * * p<0.001$. BRCA1, breast cancer gene 1; OS, overall survival; RFS, relapse-free survival; TCGA, The Cancer Genome Atlas; TIMER, Tumor Immune Estimation Resource. Color images are available online.

with worse OS in KIRP (HR=2.06, 95\% CI: 1.11-3.82, $p=0.019)$, LIHC (HR $=1.60,95 \%$ CI: $1.13-2.27, p=0.007)$, and LUAD (HR $=1.55,95 \% \mathrm{CI}: 1.16-2.08, p=0.003)$, whereas correlated with favorable OS in ESCA $(\mathrm{HR}=0.39$, 95\% CI: $0.16-0.92, p=0.027)$ and STAD (HR $=0.67,95 \%$ CI: 0.48-0.93, $p=0.016$ ) (Fig. 1B). Besides, high expression of BRCA1 was also correlated with unfavorable relapse-free survival (RFS) in LIHC (HR $=1.93$, 95\% CI: 1.39-2.70, $p<0.001$ ), whereas correlated with favorable OS in STAD (HR $=0.39,95 \%$ CI: 0.19-0.79, $p=0.007)$ (Fig. 1C).

\section{Upregulated expression of BRCA1 in HCC}

We next assessed BRCA1 transcription levels in multiple HCC cohorts from HCCDB database. Analysis of 11 HCC cohorts uncovered that mRNA expression of BRCA1 was remarkably enhanced in $\mathrm{HCC}$ tissues compared with that in normal liver tissues (Fig. 2A). We further employed Human Protein Atlas database to examine BRCA1 proteins expression in HCC and normal tissues. We found that BRCA1 protein were overexpressed in the HCC tissues compared 


\section{A}

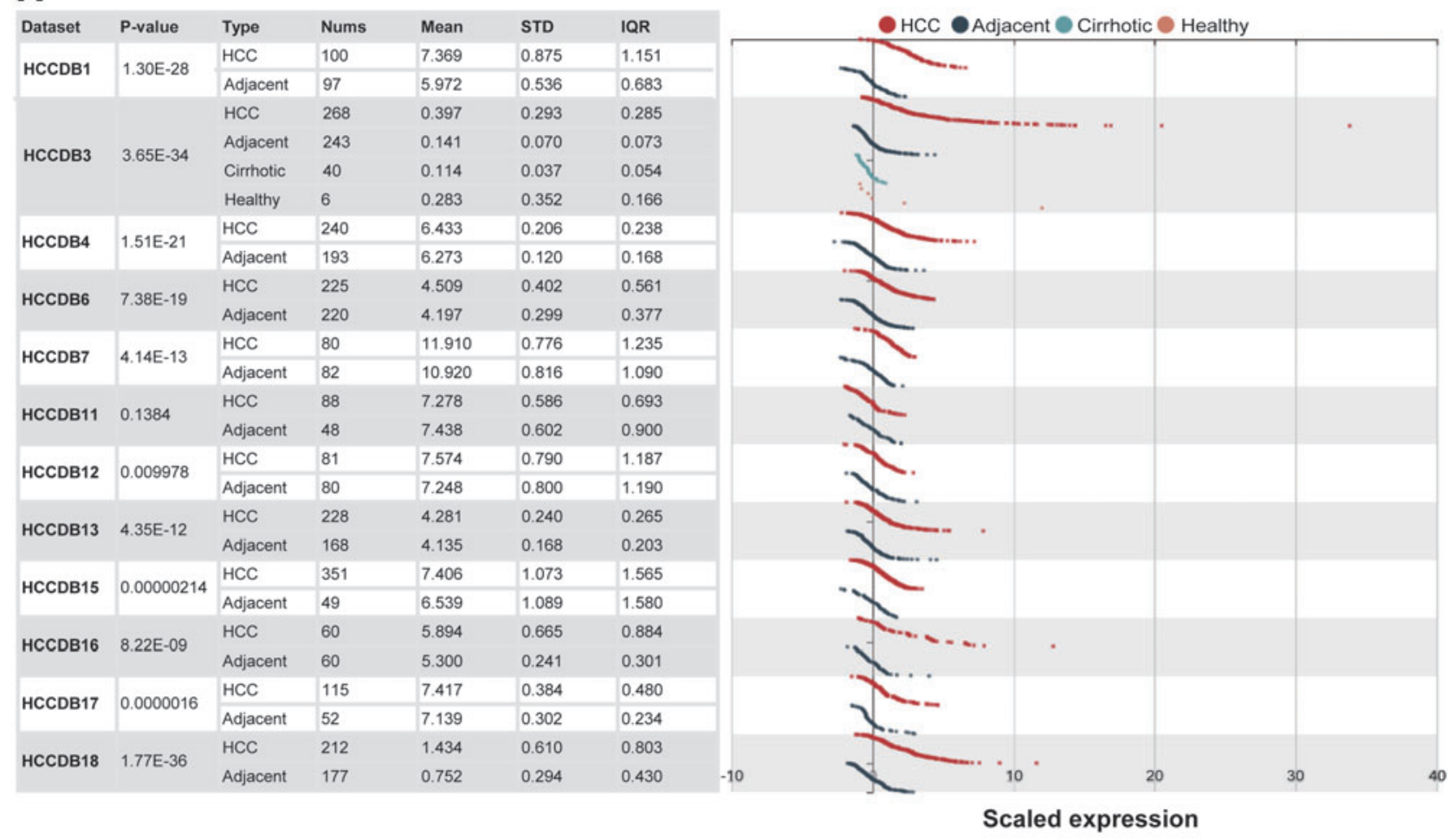

B

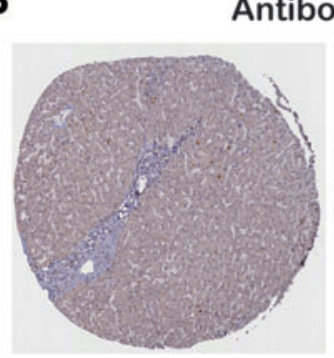

Adjacent
Antibody HPA034966

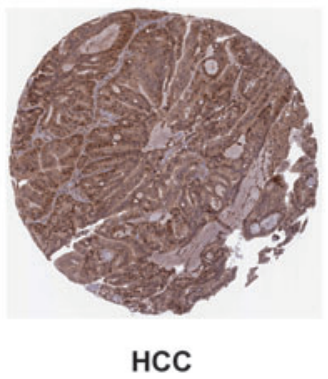

C

\begin{tabular}{lcc}
\hline Tissues & Staining & HPA034966 \\
\hline & High & 1 \\
HCC & Medium & 6 \\
& Low & 4 \\
& Not detected & 1 \\
Adjacent & $\mathrm{N}=3$ & Low \\
\hline
\end{tabular}

Antibody HPA034966

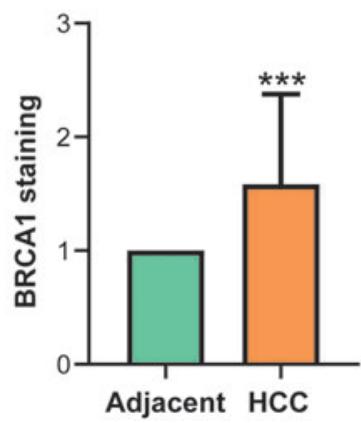

FIG. 2. BRCA1 expression level in HCC. (A) Chart and plot showing the expression of BRCA1 in tumor tissues and the adjacent normal tissues, according to $t$-test in HCCDB. (B) The protein expression of BRCA1 in HCC from Human Protein Atlas. (C) Distribution and semi-quantification of BRCA1 protein in HCC, comparing by Wilcoxon test according to samples in Human Protein Atlas. Note: $* * * p<0.001$. HCC, hepatocellular carcinoma. Color images are available online.

with that in normal liver tissues identifying by antibody HPA034966 for BRCA1 $(p<0.001$, Fig. 2B, C). Taken together, we confirmed that BRCA1 was notably upregulated in both mRNA and protein level in HCC tissues.

\section{Association of BRCA1 with clinical features and MSI status in HCC}

Considering the high expression of BRCA1 in HCC, we speculated that overexpression of BRCA1 might associate with clinical features of HCC patients. So, we analyzed the relationship between expression of BRCA1 with clinical features of $\mathrm{HCC}$ patients, including gender, age, race, $\mathrm{T}$ stage, clinical stage, and tumor grades. The baselines of HCC patients' characteristics in TCGA-LIHC dataset were shown in Supplementary Table S1.
As exhibited in Figure 3, BRCA1 expression was significantly correlated with race $(p=0.003)$, but had no relationship with gender $(p=0.253)$ and age $(p=0.092)$ in general characteristics (Fig. 3A-C). More importantly, patients who were with advanced T stages $(p=0.004)$, clinical stages $(p=0.003)$, and tumor grades $(p=0.001)$ commonly expressed higher BRCA1. The highest expression of BRCA1 was found in T4, stage 3, and grade 4, respectively (Fig. 3D-F). The reason why the mRNA expression of BRCA1 in stage 3 seemed to be higher than that in stage 4 may be due to the limited number of stage 4 patients (only six HCC patients were at stage 4).

We next assessed the correlation between BRCA1 and microsatellite instability (MSI) status, which was mainly represented by four DNA repair genes expression (MLH1, MSH2, MSH6, and PMS2). As Figure 4 exhibited, the expression of BRCA1 was positively correlated with four 
A
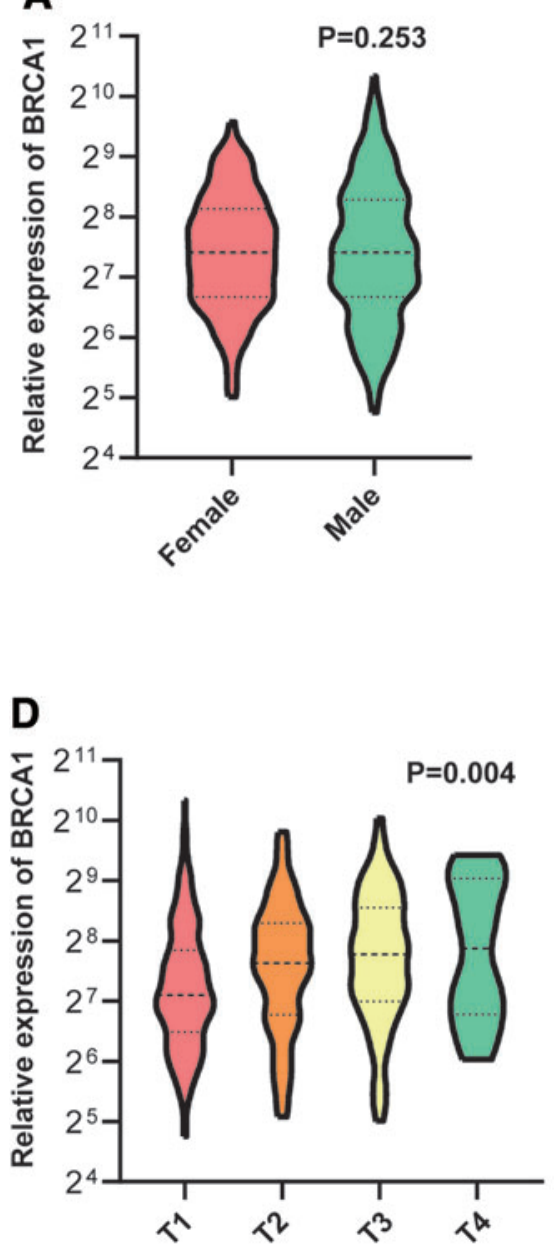

B

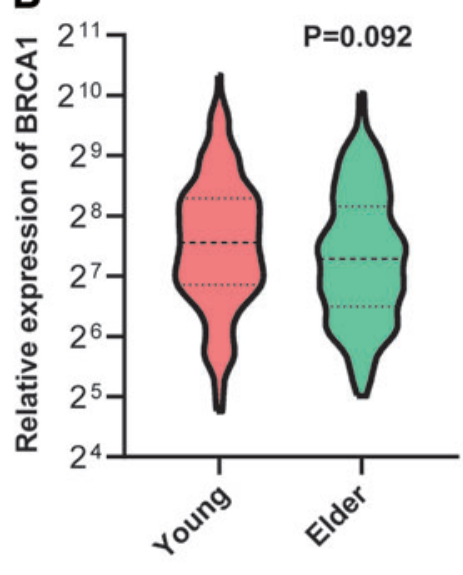

E

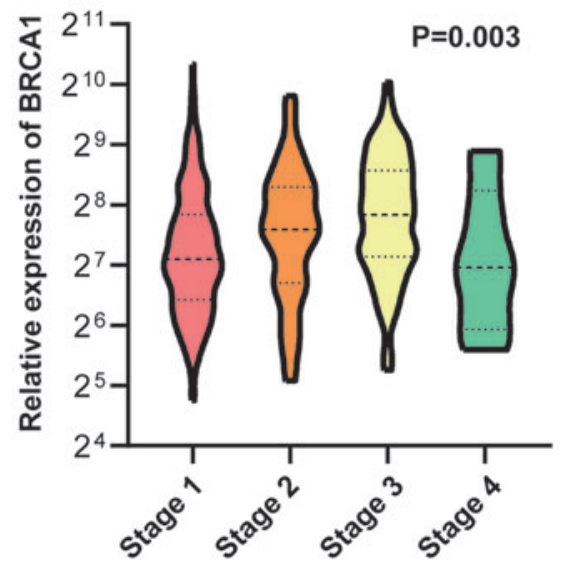

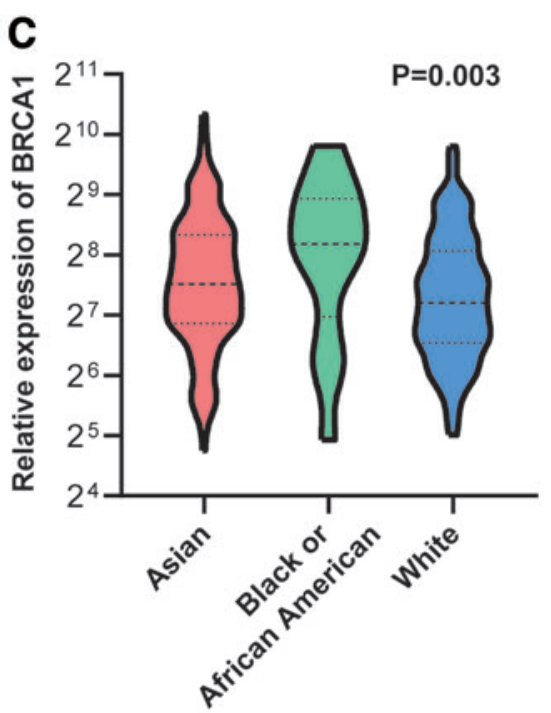

$\mathbf{F}$

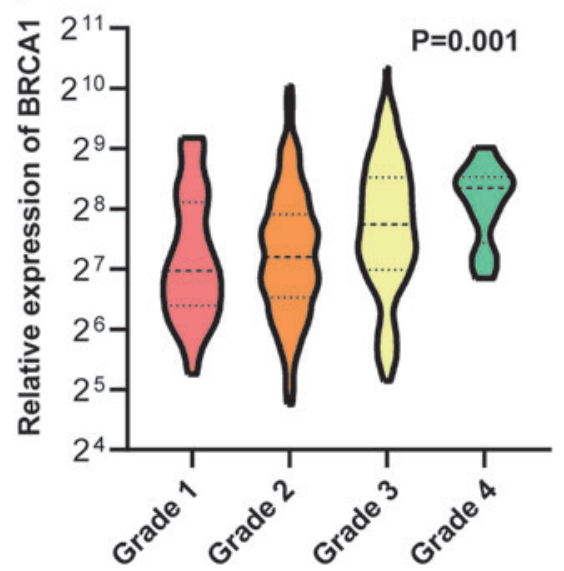

FIG. 3. BRCA1 transcriptional level in subgroups of patients with HCC. (A) Violin plot showing relative expression of BRCA1 in male or female patients. (B) Violin plot showing relative expression of BRCA1 in young ( $\leq 60$ years) or elder ( $>60$ years) patients. (C) Violin plot showing relative expression of BRCA1 in African Asian, Black or African American, White patients. (D) Violin plot showing relative expression of BRCA1 in patients in T stage 1, 2, 3, or 4. (E) Violin plot showing relative expression of BRCA1 in patients in clinical stage 1,2,3, or 4. (F) Violin plot showing relative expression of BRCA1 in patients with grade 1,2,3, or 4 tumors. Color images are available online.
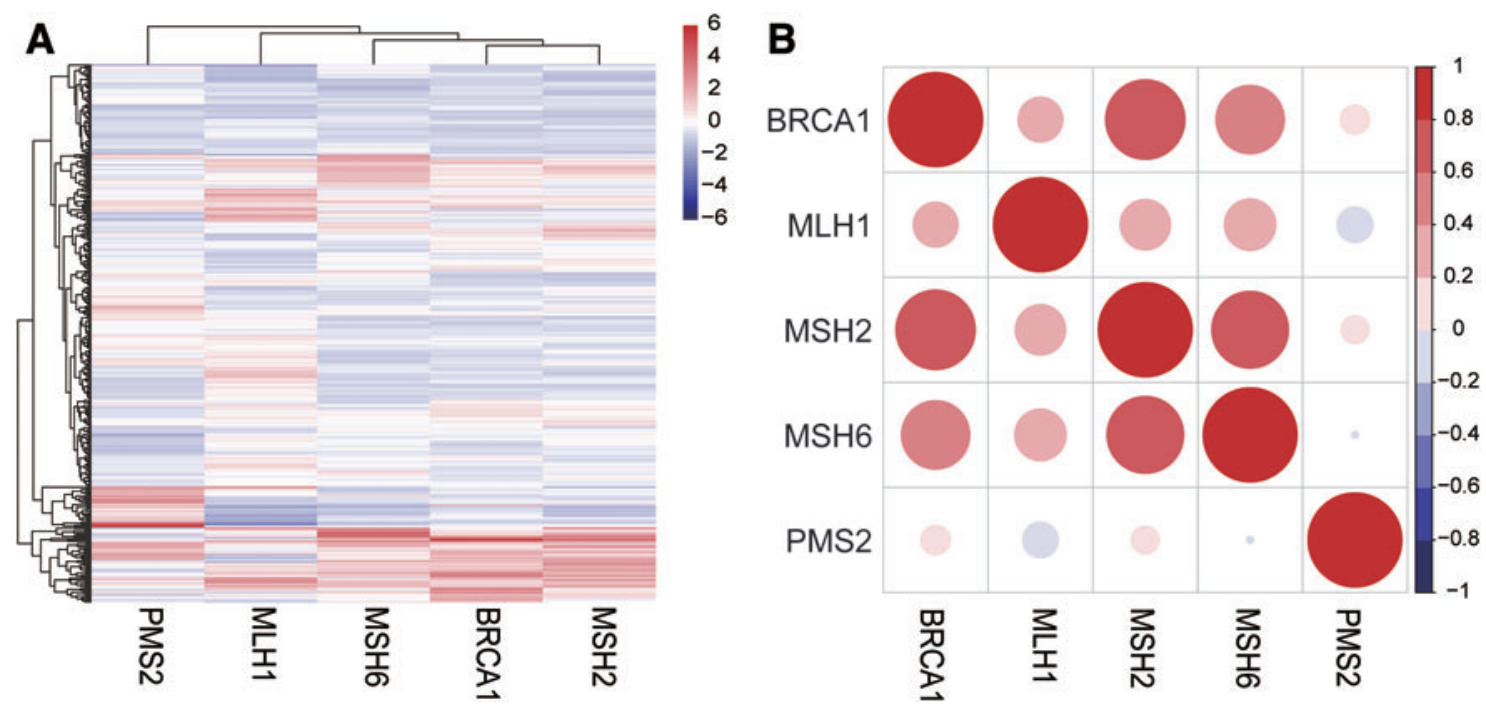

FIG. 4. Association between BRCA1 and DNA repair genes expression. (A) Correlation analysis between BRCA1 and DNA repair genes expression was summarized in the heatmap. Red: high expression, blue: low expression. (B) BRCA1 expression positively correlated with expression of DNA repair genes. Red: positive association, blue: negative association. Color images are available online. 

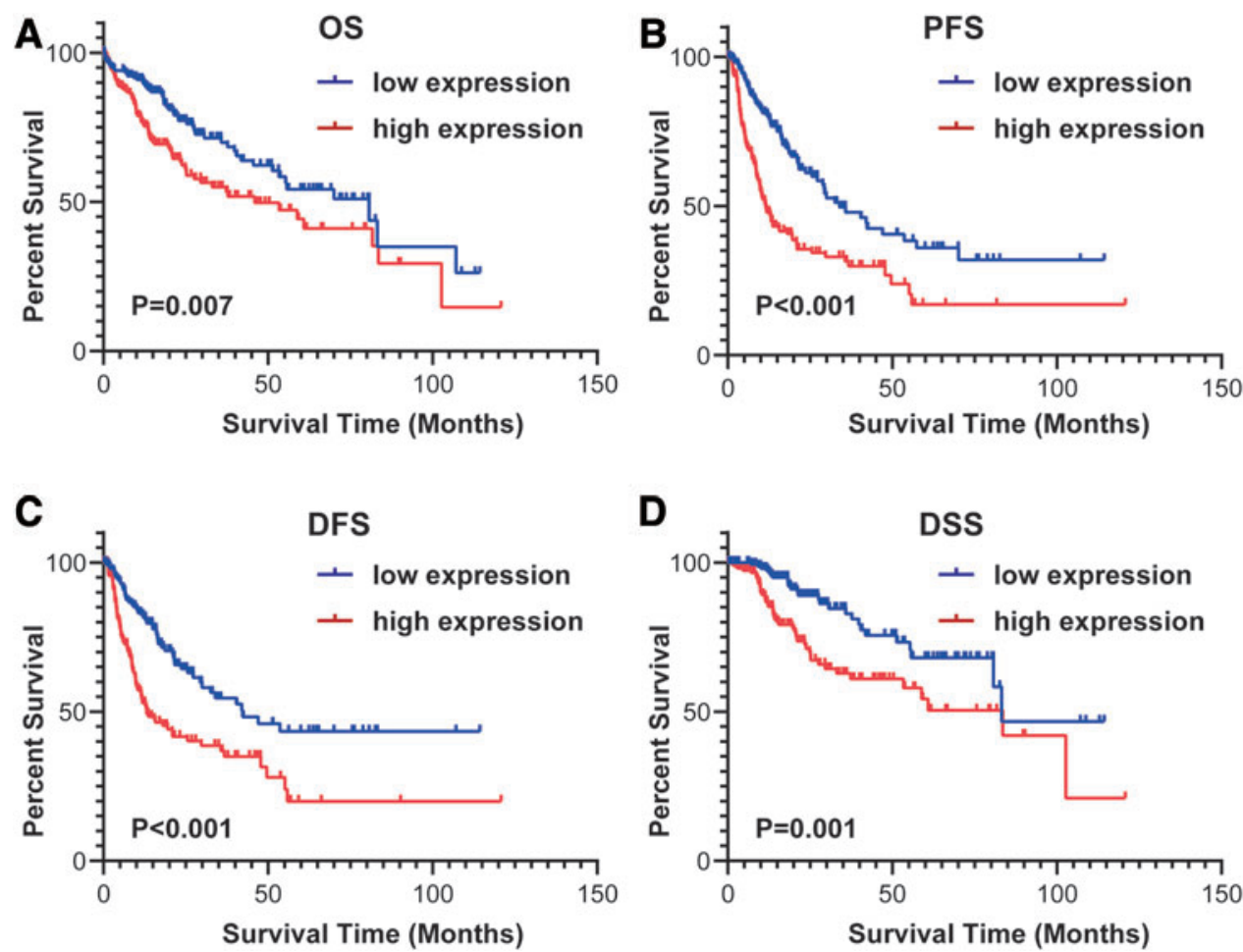

FIG. 5. The prognostic value of BRCA1 expression in HCC. (A) OS curve was plotted for HCC patients, OS of patients with high expression of BRCA1 was significantly worse than that of patients with low expression. (B) PFS curve was plotted for HCC patients, PFS of patients with high expression of BRCA1 was significantly worse than that of patients with low expression. (C) RFS curve was plotted for HCC patients, RFS of patients with high expression of BRCA1 was significantly worse than that of patients with low expression. (D) DSS curve was plotted for HCC patients,

DSS of patients with high expression of BRCA1 was significantly worse than that of patients with low expression. DSS, disease-specific survival; PFS, progression-free survival. Color images are available online.

Table 1. Univariate and Multivariate Analysis of Survival in Patients with Hepatocellular Carcinoma

\begin{tabular}{|c|c|c|c|c|c|c|}
\hline \multirow[b]{2}{*}{ Clinicopathological factors } & \multicolumn{3}{|c|}{ Univariate analysis } & \multicolumn{3}{|c|}{ Multivariate analysis } \\
\hline & $H R$ & $95 \% C I$ & $\mathrm{p}$ & $H R$ & $95 \% C I$ & $\mathrm{p}$ \\
\hline \multicolumn{7}{|l|}{ OS } \\
\hline Gender & 0.780 & $0.539-1.127$ & 0.186 & & & \\
\hline Age & 1.013 & $0.999-1.028$ & 0.075 & & & \\
\hline $\mathrm{T}$ stage & 1.657 & $1.373-1.999$ & $<\mathbf{0 . 0 0 1}$ & 1.235 & $0.649-2.347$ & 0.520 \\
\hline Clinical stage & 1.638 & $1.335-2.009$ & $<0.001$ & 1.295 & $0.669-2.505$ & 0.443 \\
\hline Histologic grade & 1.132 & $0.885-1.446$ & 0.324 & & & \\
\hline BRCA1 expression & 1.645 & $1.143-2.369$ & 0.007 & 1.456 & $0.992-2.138$ & 0.055 \\
\hline \multicolumn{7}{|l|}{ PFS } \\
\hline Gender & 0.995 & $0.719-1.378$ & 0.977 & & & \\
\hline Age & 0.996 & $0.984-1.008$ & 0.478 & & & \\
\hline $\mathrm{T}$ stage & 1.603 & $1.365-1.883$ & $<0.001$ & 0.866 & $0.497-1.511$ & 0.613 \\
\hline Clinical stage & 1.649 & $1.380-1.920$ & $<0.001$ & 1.810 & $1.026-3.193$ & 0.040 \\
\hline Histologic grade & 1.130 & $0.920-1.388$ & 0.244 & & & \\
\hline BRCA1 expression & 2.151 & $1.574-2.940$ & $<0.001$ & 1.964 & $1.416-2.725$ & $<\mathbf{0 . 0 0 1}$ \\
\hline \multicolumn{7}{|l|}{ DFS } \\
\hline Gender & 1.184 & $0.818-1.714$ & 0.370 & & & \\
\hline Age & 0.996 & $0.983-1.010$ & 0.610 & & & \\
\hline $\mathrm{T}$ stage & 1.653 & $1.364-2.004$ & $<\mathbf{0 . 0 0 1}$ & 0.980 & $0.470-2.043$ & 0.958 \\
\hline Clinical stage & 1.677 & $1.363-2.063$ & $<0.001$ & 1.661 & $0.786-3.510$ & 0.184 \\
\hline Histologic grade & 1.214 & $0.965-1.527$ & 0.099 & & & \\
\hline BRCA1 expression & 2.137 & $1.504-3.038$ & $<0.001$ & 2.047 & $1.423-2.945$ & $<0.001$ \\
\hline \multicolumn{7}{|l|}{ DSS } \\
\hline Gender & 0.852 & $0.527-1.372$ & 0.510 & & & \\
\hline Age & 1.003 & $0.985-1.021$ & 0.748 & & & \\
\hline $\mathrm{T}$ stage & 2.005 & $1.577-2.548$ & $<0.001$ & 0.866 & $0.558-2.311$ & 0.725 \\
\hline Clinical stage & 2.020 & $1.542-2.645$ & $<0.001$ & 1.701 & $0.825-3.510$ & 0.150 \\
\hline Histologic grade & 1.125 & $0.820-1.544$ & 0.464 & & & \\
\hline BRCA1 expression & 2.205 & $1.361-3.570$ & $<0.001$ & 1.936 & $1.144-3.275$ & $<\mathbf{0 . 0 0 1}$ \\
\hline
\end{tabular}

Bold means statistical significance.

BRCA1, breast cancer gene 1; CI, confidence interval; DFS, disease-free survival; DSS, disease-specific survival; HR, hazard ratio; OS, overall survival; PFS, progression-free survival. 
repair genes, and the most obvious correlation was found between BRCA1 and MSH2 (Fig. 4A, B).

\section{Prognostic value of BRCA1 expression in HCC}

Furthermore, we validated the prognostic value of BRCA1 as well. Original TCGA dataset curated survival data included four endpoints, and recommends the use of the endpoints of OS, progression-free survival (PFS), disease-free survival (DFS), and disease-specific survival (DSS) for each TCGA cancer type (Liu et al., 2018). As shown in Figure 5, high mRNA expression of BRCA1 was remarkably related to poor OS $(p=0.007)$, PFS $(p<0.001)$, DFS $(p<0.001)$, and DSS $(p=0.001)$ of HCC patients (Fig. 5A-D). Besides, in HCCDB18 cohort, high mRNA expression of BRCA1 was also notably correlated with poor OS $(p<0.001$, Supplementary Fig. S1).

To further evaluate whether BRCA1 expression is an independent prognostic factor for patients with $\mathrm{HCC}$, the

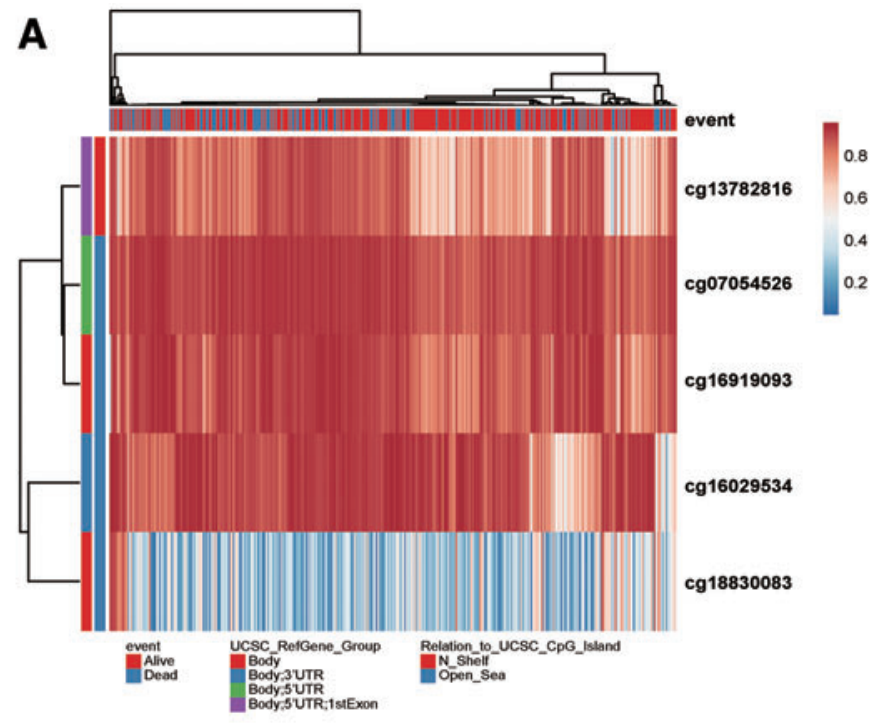

B

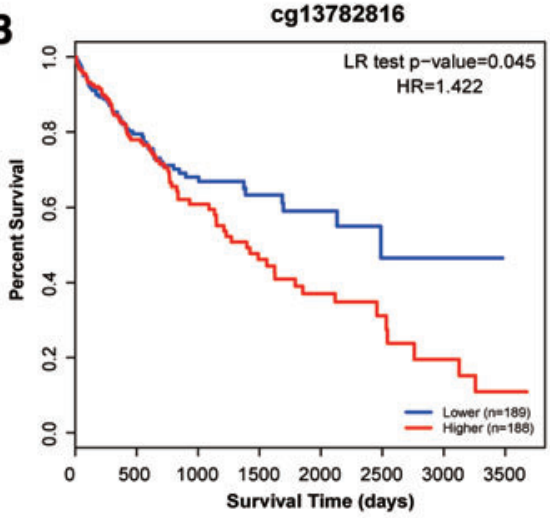

C

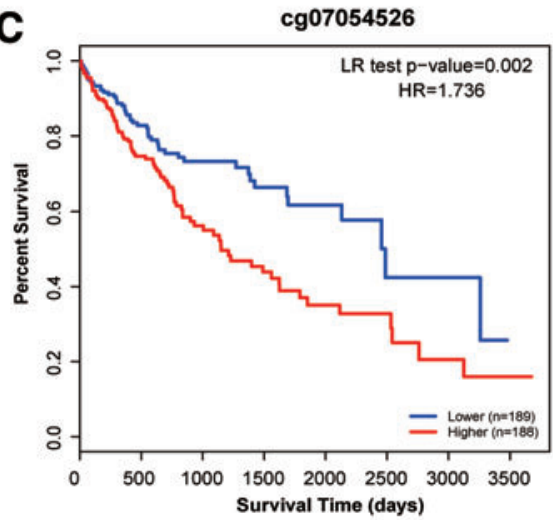

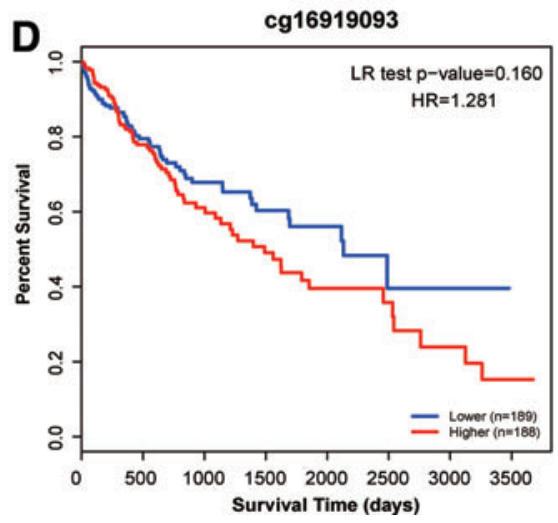
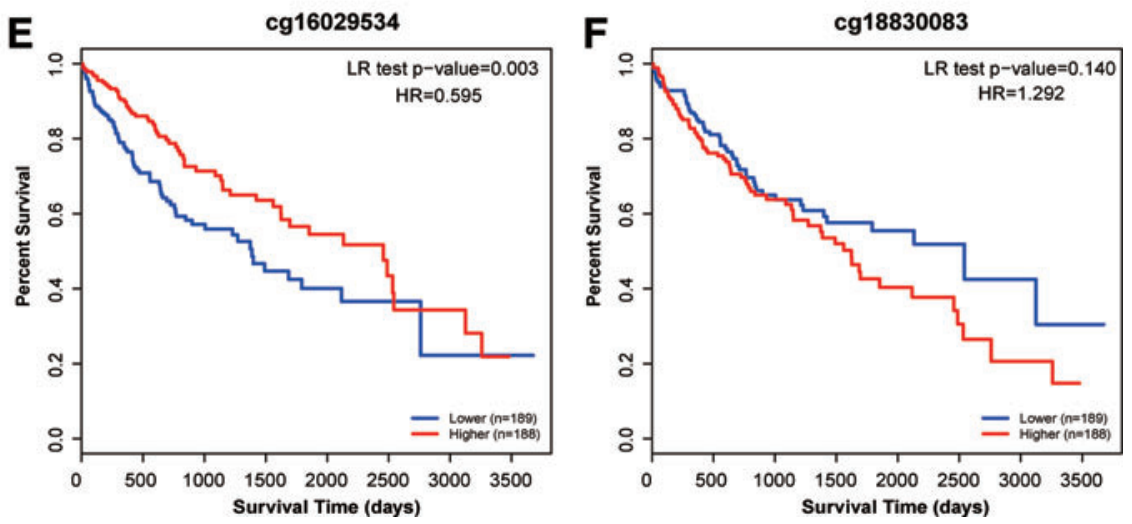

FIG. 6. DNA methylation sites of BRCA1 and their prognostic values in HCC. (A) The DNA methylation clustered expression of BRCA1. (B) OS curve of cg13782816 in HCC patients in MethSurv. (C) OS curve of cg07054526 in HCC patients in MethSurv. (D) OS curve of cg16919093 in HCC patients in MethSurv. (E) OS curve of cg16029534 in HCC patients in MethSurv. (F) OS curve of cg18830083 in HCC patients in MethSurv. Color images are available online. 
Cox regression model was applied for univariate and multivariate survival analyses. Univariate analysis exhibited that BRCA1 expression status in addition to $\mathrm{T}$ stage and clinical stage were associated with OS $(\mathrm{HR}=1.645,95 \%$ CI: $1.143-$ 2.369, $p=0.007)$, PFS $(\mathrm{HR}=2.151,95 \% \mathrm{CI}: 1.574-2.940$, $p<0.001)$, DFS (HR $=2.137,95 \%$ CI: $1.504-3.038, p<0.001)$, and DSS $(\mathrm{HR}=2.205,95 \% \mathrm{CI}: 1.361-3.570, p<0.001)$ (Table 1). In multivariate analysis, we confirmed that BRCA1 expression was an independent prognostic indicator for PFS (HR $=1.964,95 \%$ CI: $1.416-2.725, p<0.001)$, DFS $(\mathrm{HR}=2.047,95 \%$ CI: $1.423-2.945, p<0.001)$, and DSS $(\mathrm{HR}=1.936,95 \% \mathrm{CI}: 1.144-3.275, p<0.001)$ in HCC patients (Table 1).

\section{BRCA1 DNA methylation and their prognostic values in $\mathrm{HCC}$}

The DNA methylation levels of each CpG in BRCA1 were analyzed and displayed by MethSurv (Fig. 6A). Five CpGs sites were found in BRCA1 gene, including cg13782816, cg07054526, cg16919093, cg16029534, and cg18830083 (Fig. 5B-F). Furthermore, the prognostic value of each $\mathrm{CpG}$ site was explored. A total of three prognostic-related $\mathrm{CpGs}$ were identified in BRCA1 gene. As shown in Figure 6, high DNA methylation levels of $\operatorname{cg} 13782816(\mathrm{HR}=1.422$, $p=0.045)$ and $\operatorname{cg} 07054526(\mathrm{HR}=1.736, p=0.002)$ were highly correlated with poor OS in HCC patients (Fig. 6B, C), while low DNA methylation levels of cg16029534 $(\mathrm{HR}=0.595, p=0.003)$ were correlated with poor OS in HCC patients (Fig. 6E).

\section{Genetic alterations in BRCA1 in HCC}

CNA is a usual genetic alteration and is associated with oncogenesis and development of cancers via regulating the expression of tumor-related genes (Thomas et al., 2015; Bigagli et al., 2016; Choi et al., 2017). From the fact that BRCA1 was dysregulated in HCC tissues, we speculated that CNA may be a critical factor in regulating the transcriptional levels of BRCA1. We analyzed genetic alterations in BRCA1 gene and their correlations with its mRNA expression. A low amplification rate of BRCA1 was found in HCC patients. However, although copy gain (gain and amplification) of BCRA1 was not common, it was still remarkably correlated with upregulated BRCA1 mRNA levels compared with the copy-neutral (diploid) and copy-loss (shallow deletion) samples $(p<0.001$, Fig. 7A).

As we all know, $\sim 5-10 \%$ occurrence of breast cancers are due to genetic mutations, and the most commonly tested gene is BRCA1 (Kwong et al., 2016). Thus, we next analyzed genetic alterations in BRCA1 in HCC samples. A low mutation rate of BRCA1 was observed in HCC patients. In the 348 sequenced $\mathrm{HCC}$ patients, the genetic alteration was found in only $11 \mathrm{HCC}$ samples (Fig. 7B).

\section{Correlation between BRCA1 expression and immune cells infiltration and markers of different subsets in HCC}

The prognosis of patients in several cancers is affected by the quantity and activity status of TIICs (Chen et al., 2017; Almangush et al., 2018; Denkert et al., 2018). Therefore, we investigated the association between BRCA1 expression and the abundance of TIICs in HCC based on TIMER database. The results exhibited that BRCA1 expression had significant correlations with tumor purity (percentage of malignant cells in a tumor tissue) (cor $=0.179, p<0.001)$ and significant correlations with the multiple immune cells abundance, including B cells $(\mathrm{cor}=0.355, p<0.001), \mathrm{CD} 8+\mathrm{T}$ cells $(\mathrm{cor}=$ $0.281, p<0.001), \mathrm{CD} 4+\mathrm{T}$ cells $(\mathrm{cor}=0.185, p<0.001)$, neutrophils (cor $=0.319, p<0.001)$, macrophages (cor $=0.320$, $p<0.001$ ), and DCs (cor $=0.361, p<0.001$ ) (Fig. 8).

Furthermore, we assessed the correlation between BRCA1 expression and the status of TIICs based on the expression levels of immune marker in HCC tissues via TIMER database. The immune cells analyzed in HCC tissues included CD8+ $\mathrm{T}$ cells, B cells, tumor-associated macrophages (TAMs), monocytes, M1 and M2 macrophages, neutrophils, DCs, and natural killer (NK) cells. Besides, different subsets of $\mathrm{T}$ cells, namely, T helper 1 (Th1), Th2, follicular helper $\mathrm{T}$ (Tfh), Th17, regulatory $\mathrm{T}$ (Tregs), and exhausted $\mathrm{T}$ cells were also included. Given tumor purity affected the analysis of immune cell infiltration, the correlation analysis was adjusted for tumor purity.

Analysis of the TIMER database exhibited that BRCA1 expression in HCC tissues prominently correlated with the expression of marker genes of CD8+ T cells, T cells (general), B cells, monocyte, TAMs, neutrophils, NK cells, DCs, Th1 cells, Th2 cells, Tfh cells, Th17 cells, Treg cells, and $\mathrm{T}$ cell exhaustion. However, BRCA1 expression exhibited
A
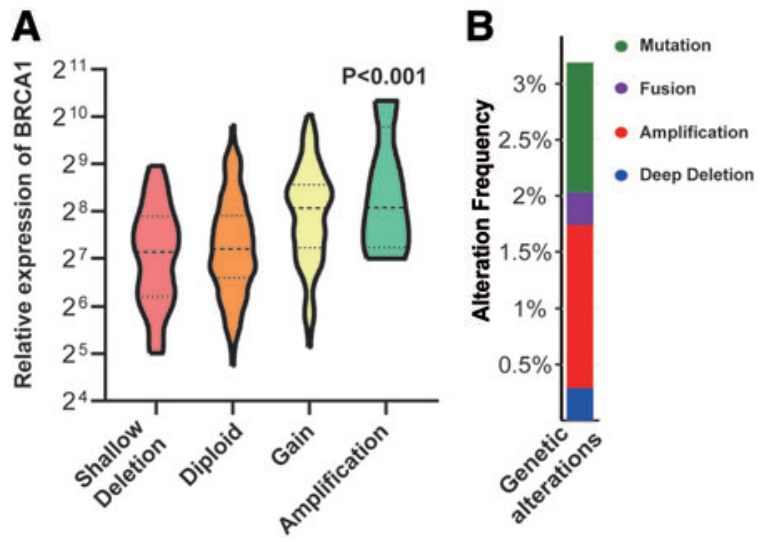

FIG. 7. Genetic alterations of BRCA1 in HCC. (A) Copy gain (gain and amplification) of BRCA1 was associated with notably upregulated BRCA1 mRNA levels compared with the copy-neutral (diploid) and copyloss (shallow deletion) cases. Shallow deletion: 33 cases, diploid: 221 cases, gain: 88 cases, amplification: 5 cases. (B) The proportion and distribution of samples with genetic alterations of BRCA1 in HCC. Color images are available online. 


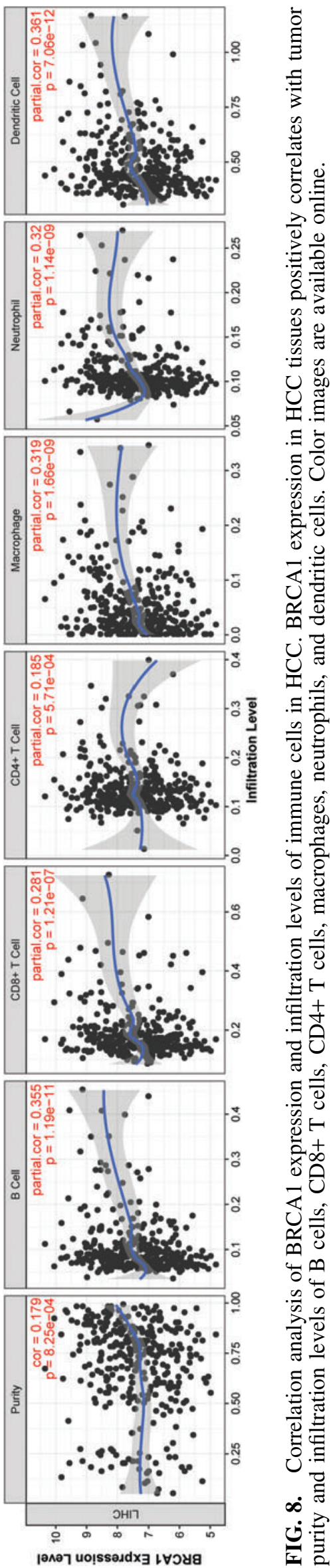

no correlation with the expression of marker genes for M1 and M2 macrophage in HCC (Table 2).

\section{Discussion}

BRCA1 is a star gene with tumor-suppressive roles in multiple cancers, especially in breast and ovarian cancer. Mutations in BRCA1 give rise to about 50\% of inherited breast cancer and are correlated with increased risk of breast and ovarian cancer oncogenesis and worse prognosis in prostate cancer (King et al., 2003; Agalliu et al., 2009). BRCA1 mRNA and protein are downregulated by epigenetic modifications in about 30\% sporadic breast cancers and 70\% ovarian cancer, but also occur in gastric, lung, and other cancers (Taron et al., 2004; Chen et al., 2013; De Luca and De Siervi, 2016).

BRCA1 also functions as a monitor to predict the response to chemotherapy. Patients with reduced BRCA1 expression exhibit better OS when receiving platinum-based adjuvant chemotherapy (Chen et al., 2013). Xu et al. (2018) revealed that BRCA1 transcriptional expression could be treated as a biomarker in breast cancer to predict curative effect in BRCA1/2 mutation-negative patients who received anthracycline-based neoadjuvant. Besides, low BRCA1/2-expression reduces the capacity of DNA damage repair in tissues, which enhances platinum-sensitivity in ovarian cancer (Tsibulak et al., 2018).

In addition to serving as inherited and prognostic markers in cancers, BRCA1 also affects cancer progression. In breast cancer, the breast cancer related gene genetrinucleotiderepeat-containing 9 (TNRC9) has been confirmed correlated with disease susceptibility (Chen et al., 2016). Shan et al. (2013) found that TNRC9 is often overexpressed in breast cancer and overexpression of TNRC9 promotes cell proliferation, migration by downregulating BRCA1 expression through altering its methylation status. Besides, upregulation of BRCA1 could notably increase expression of PIG3 in cells with intact P53, thus regulating PIG3-mediated apoptosis in breast cancer (Zhang et al., 2015). BRCA1 also suppresses androgen receptor-induced proliferation of breast cancer cells through activating SIRT1 (Zhang et al., 2016). However, there is rare research on the molecular mechanisms of BRCA1 affecting other cancer progressions excepting breast cancer.

Although lots of studies focused on the molecular functions and clinical associations of BRCA1, its exact prognostic roles in cancers have not been explored. To gain more novel insights into the potential prognostic potentials of BRCA1, we performed the systematic bioinformatics analysis of public data. This study demonstrated that BRCA1 mRNA levels were associated with prognosis of several human cancers, and the most notable prognostic value was found in HCC. Considering that overexpressed BRAC1 was associated with both OS and RFS in HCC in pan-cancer analysis, we speculated BRCA1 might be a novel biomarker of poor prognosis in HCC. Thus, we further conducted an in-depth analysis of BRCA1 to elucidate its potential role in HCC.

As result, the upregulation of BRCA1 was correlated with poor prognosis, clinical features, and MSI status. One possible explanation for association between high BRCA1 expression and poor prognosis, high BRCA1 would protect HCC from further DNA damage, which might promote resistance to chemotherapeutics. In tongue squamous cell carcinoma, lncRNA CISAL inhibited BRCA1 transcription by forming a tertiary structure at its promoter and associated 
Table 2. Correlation Analysis Between BRCA1 and Related Gene Markers of Immune Cells

\begin{tabular}{|c|c|c|c|c|c|}
\hline \multirow[b]{2}{*}{ Description } & \multirow[b]{2}{*}{ Gene markers } & \multicolumn{2}{|c|}{ None } & \multicolumn{2}{|c|}{ Purity } \\
\hline & & Core & $\mathrm{p}$ & Core & $\mathrm{p}$ \\
\hline \multirow{2}{*}{ CD8+ $\mathrm{T}$ cell } & CD8A & 0.027 & 0.609 & 0.129 & 0.016 \\
\hline & CD8B & 0.043 & 0.404 & 0.144 & 0.007 \\
\hline \multirow[t]{3}{*}{$\mathrm{T}$ cell (general) } & CD3D & 0.036 & 0.486 & 0.142 & 0.008 \\
\hline & CD3E & -0.046 & 0.375 & 0.074 & 0.171 \\
\hline & CD2 & -0.029 & 0.583 & 0.093 & 0.085 \\
\hline \multirow[t]{2}{*}{ B cell } & CD19 & 0.135 & 0.009 & 0.209 & $<0.001$ \\
\hline & CD79A & 0.019 & 0.713 & 0.132 & 0.014 \\
\hline \multirow[t]{2}{*}{ Monocyte } & CD86 & 0.071 & 0.173 & 0.214 & $<0.001$ \\
\hline & CSF1R & -0.041 & 0.434 & 0.079 & 0.142 \\
\hline \multirow[t]{3}{*}{ TAM } & CCL2 & -0.135 & 0.009 & -0.061 & 0.259 \\
\hline & CD68 & -0.054 & 0.303 & 0.031 & 0.572 \\
\hline & IL10 & 0.050 & 0.335 & 0.159 & 0.003 \\
\hline \multirow[t]{3}{*}{ M1 macrophage } & NOS2 & -0.095 & 0.067 & -0.078 & 0.147 \\
\hline & IRF5 & 0.093 & 0.073 & 0.088 & 0.101 \\
\hline & PTGS2 & -0.066 & 0.203 & 0.046 & 0.392 \\
\hline \multirow[t]{3}{*}{ M2 macrophage } & CD163 & -0.023 & 0.665 & 0.082 & 0.129 \\
\hline & VSIG4 & -0.006 & 0.903 & 0.095 & 0.078 \\
\hline & MS4A4A & -0.081 & 0.121 & 0.024 & 0.654 \\
\hline \multirow[t]{3}{*}{ Neutrophils } & CEACAM8 & 0.081 & 0.118 & 0.110 & 0.042 \\
\hline & ITGAM & 0.200 & $<\mathbf{0 . 0 0 1}$ & 0.299 & $<0.001$ \\
\hline & CCR7 & -0.092 & 0.075 & 0.025 & 0.641 \\
\hline \multirow[t]{7}{*}{ Natural killer cell } & KIR2DL1 & -0.085 & 0.103 & -0.111 & 0.040 \\
\hline & KIR2DL3 & 0.068 & 0.191 & 0.121 & 0.025 \\
\hline & KIR2DL4 & 0.129 & 0.013 & 0.164 & 0.002 \\
\hline & KIR3DL1 & -0.014 & 0.795 & 0.000 & 0.996 \\
\hline & KIR3DL2 & 0.027 & 0.610 & 0.070 & 0.194 \\
\hline & KIR3DL3 & -0.004 & 0.940 & 0.000 & 0.993 \\
\hline & KIR2DS4 & -0.038 & 0.464 & -0.051 & 0.350 \\
\hline \multirow[t]{7}{*}{ Dendritic cell } & HLA-DPB1 & -0.062 & 0.234 & 0.041 & 0.448 \\
\hline & HLA-DQB1 & -0.050 & 0.339 & 0.040 & 0.455 \\
\hline & HLA-DRA & -0.019 & 0.718 & 0.088 & 0.104 \\
\hline & HLA-DPA1 & -0.044 & 0.399 & 0.067 & 0.211 \\
\hline & $\mathrm{CD} 1 \mathrm{C}$ & -0.068 & 0.189 & 0.020 & 0.717 \\
\hline & NRP1 & 0.027 & 0.602 & 0.071 & 0.186 \\
\hline & ITGAX & 0.068 & 0.194 & 0.190 & $<\mathbf{0 . 0 0 1}$ \\
\hline \multirow[t]{5}{*}{ Th1 cell } & TBX21 & -0.094 & 0.072 & -0.008 & 0.887 \\
\hline & STAT4 & 0.023 & 0.663 & 0.085 & 0.115 \\
\hline & STAT1 & 0.186 & $<\mathbf{0 . 0 0 1}$ & 0.247 & $<0.001$ \\
\hline & IFNG & 0.141 & 0.007 & 0.229 & $<0.001$ \\
\hline & TNF & 0.053 & 0.308 & 0.176 & 0.001 \\
\hline \multirow[t]{4}{*}{ Th2 cell } & GATA3 & -0.053 & 0.307 & 0.061 & 0.259 \\
\hline & STAT6 & -0.232 & $<0.001$ & -0.250 & $<0.001$ \\
\hline & STAT5A & 0.051 & 0.332 & 0.137 & 0.011 \\
\hline & IL13 & 0.117 & 0.025 & 0.131 & 0.015 \\
\hline \multirow[t]{2}{*}{ Tfh cell } & BCL6 & -0.028 & 0.593 & -0.023 & 0.666 \\
\hline & IL21 & 0.135 & 0.009 & 0.190 & $<0.001$ \\
\hline Th17 cell & STAT3 & -0.031 & 0.557 & 0.024 & 0.652 \\
\hline & IL17A & 0.015 & 0.773 & 0.032 & 0.555 \\
\hline Treg cell & FOXP3 & 0.084 & 0.106 & 0.156 & 0.004 \\
\hline & CCR8 & 0.227 & $<\mathbf{0 . 0 0 1}$ & 0.329 & $<0.001$ \\
\hline & STAT5B & 0.051 & 0.326 & 0.033 & 0.545 \\
\hline & TGFB1 & -0.043 & 0.405 & 0.051 & 0.344 \\
\hline $\mathrm{T}$ cell exhaustion & PDCD1 & 0.094 & 0.069 & 0.185 & $<0.001$ \\
\hline & CTLA4 & 0.135 & 0.009 & 0.249 & $<0.001$ \\
\hline & LAG3 & 0.095 & 0.066 & 0.142 & 0.008 \\
\hline & HAVCR2 & 0.092 & 0.077 & 0.238 & $<0.001$ \\
\hline & GZMB & -0.044 & 0.398 & 0.018 & 0.734 \\
\hline
\end{tabular}

Bold means statistical significance.

TAM, tumor-associated macrophage; Tfh, follicular helper T; Th1, T helper 1; Tregs, regulatory T. 
favorable neoadjuvant chemosensitivity (Fan et al., 2020), which was consistent with our conjecture. Wang et al. (2018) performed a systematic analysis to uncover the expression profiles and prognostic values of BRCA1 in digestive system cancers. In HCC, BRCA1 is found to be mostly localized in the nucleus, and high nucleus BRCA1 expression is associated with poor survival (Wang et al., 2018). These results highly supported our findings in the current research.

As we all know, mutations in BRCA1 are highly associated with breast and ovarian cancer risk, so we also analyzed its genetic alterations in HCC. A low mutation rate of BRCA1, about $3.2 \%$, was found in HCC patients. In the 348 sequenced HCC patients, genetic alteration was found in only $11 \mathrm{HCC}$ patients. However, in TCGA-BRCA cohort, about 2.7\% (27/984) mutations were found in breast cancer, which was similar to that in HCC. However, whether BRCA1 mutations were associated with HCC clinical characteristics and prognosis could not be explored due to the limited sample sizes in TCGA-LIHC dataset.

The current research also demonstrated that BRCA1 expression correlated with the infiltrating status of immune cells in HCC. Our results exhibited that BRCA1 expression had significant correlations with tumor purity and notable correlations with the dominant immune cells infiltration levels, including B cells, CD8+ T cells, CD4+ T cells, neutrophils, macrophages, and DCs. Several studies confirm that genomic instability led to increasing expression of checkpoint inhibitor molecules and infiltration of immune cells (Giannakis et al., 2016; Le et al., 2017).

Interestingly, BRCA1-deficient breast cancers are observed to be correlated with higher expression of PD-L1 and PD-1, and increased abundance of TIICs involved in adaptive immune response (Wen and Leong, 2019). However, contrary to breast cancer, BRCA1 expression was positively associated with infiltration levels of immune cells, suggesting an opposite role of BRCA1 in HCC compared with breast cancer. Whether it is the error of bioinformatics research or the difference between various tumor species, we will explore in further research the different roles of BRCA1 in breast cancer and HCC.

\section{Conclusion}

To sum up, this research offers multilevel clues on the significance of BRCA1 in oncogenesis and development and its potential as a novel indicator in HCC. Our results uncover that BRCA1 is upregulated in $\mathrm{HCC}$ and overexpressed BRCA1 is associated with advanced stages and poor prognosis. Furthermore, our results reveal that BRCA1 is tightly associated with immune cell infiltration in HCC. Overall, these findings lay the foundations for subsequent functional studies to confirm the important role of BRCA1 in HCC.

\section{Disclosure Statement}

No competing financial interests exist.

\section{Funding Information}

This work was founded by the Natural Science Foundation of Jiangsu Province (BE2017626).

\section{Supplementary Material}

Supplementary Table S1

Supplementary Figure S1

\section{References}

Agalliu, I., Gern, R., Leanza, S., and Burk, R.D. (2009). Associations of high-grade prostate cancer with BRCA1 and BRCA2 founder mutations. Clin Cancer Res 15, 1112-1120. Almangush, A., Ruuskanen, M., Hagstrom, J., Hirvikoski, P., Tommola, S., Kosma, V.M., et al. (2018). Tumor-infiltrating lymphocytes associate with outcome in nonendemic nasopharyngeal carcinoma: a multicenter study. Hum Pathol 81, 211-219.

Bigagli, E., De Filippo, C., Castagnini, C., Toti, S., Acquadro, F., Giudici, F., et al. (2016). DNA copy number alterations, gene expression changes and disease-free survival in patients with colorectal cancer: a 10 year follow-up. Cell Oncol (Dordr) 39, 545-558.

Cerami, E., Gao, J., Dogrusoz, U., Gross, B.E., Sumer, S.O., Aksoy, B.A., et al. (2012). The cBio cancer genomics portal: an open platform for exploring multidimensional cancer genomics data. Cancer Discov 2, 401-404.

Chen, T.H., Zhang, Y.C., Tan, Y.T., An, X., Xue, C., Deng, Y.F., et al. (2017). Tumor-infiltrating lymphocytes predict prognosis of breast cancer patients treated with anti-Her-2 therapy. Oncotarget 8, 5219-5232.

Chen, W., Wang, J., Li, X., Li, J., Zhou, L., Qiu, T., et al. (2013). Prognostic significance of BRCA1 expression in gastric cancer. Med Oncol 30, 423.

Chen, Y., Shi, C., and Guo, Q. (2016). TNRC9 rs12443621 and FGFR2 rs2981582 polymorphisms and breast cancer risk. World J Surg Oncol 14, 50.

Choi, W., Ochoa, A., McConkey, D.J., Aine, M., Hoglund, M., Kim, W.Y., et al. (2017). Genetic alterations in the molecular subtypes of bladder cancer: illustration in the cancer genome atlas dataset. Eur Urol 72, 354-365.

De Luca, P., and De Siervi, A. (2016). Critical role for BRCA1 expression as a marker of chemosensitivity response and prognosis. Front Biosci (Elite Ed) 8, 72-83.

De Stefano, F., Chacon, E., Turcios, L., Marti, F., and Gedaly, R. (2018). Novel biomarkers in hepatocellular carcinoma. Dig Liver Dis 50, 1115-1123.

Denkert, C., von Minckwitz, G., Darb-Esfahani, S., Lederer, B., Heppner, B.I., Weber, K.E., et al. (2018). Tumour-infiltrating lymphocytes and prognosis in different subtypes of breast cancer: a pooled analysis of 3771 patients treated with neoadjuvant therapy. Lancet Oncol 19, 40-50.

Ding, J., and Wang, H. (2014). Multiple interactive factors in hepatocarcinogenesis. Cancer Lett 346, 17-23.

Fan, S., Tian, T., Lv, X., Lei, X., Yang, Z., Liu, M., et al. (2020). lncRNA CISAL inhibits BRCA1 transcription by forming a tertiary structure at its promoter. iScience 23, 100835.

Gao, J., Aksoy, B.A., Dogrusoz, U., Dresdner, G., Gross, B., Sumer, S.O., et al. (2013). Integrative analysis of complex cancer genomics and clinical profiles using the cBioPortal. Sci Signal 6, pl1.

Giannakis, M., Mu, X.J., Shukla, S.A., Qian, Z.R., Cohen, O., Nishihara, R., et al. (2016). Genomic correlates of immunecell infiltrates in colorectal carcinoma. Cell Rep 17, 1206.

Gu, Y., Li, X., Bi, Y., Zheng, Y., Wang, J., Li, X., et al. (2020). CCL14 is a prognostic biomarker and correlates with immune infiltrates in hepatocellular carcinoma. Aging (Albany NY) 12, 784-807.

Hedau, S., Batra, M., Singh, U.R., Bharti, A.C., Ray, A., and Das, B.C. (2015). Expression of BRCA1 and BRCA2 proteins and their correlation with clinical staging in breast cancer. J Cancer Res Ther 11, 158-163.

King, M.C., Marks, J.H., Mandell, J.B., and New York Breast Cancer Study, G. (2003). Breast and ovarian cancer risks 
due to inherited mutations in BRCA1 and BRCA2. Science 302, 643-646.

Kwong, A., Shin, V.Y., Ho, J.C., Kang, E., Nakamura, S., Teo, S.H., et al. (2016). Comprehensive spectrum of BRCA1 and BRCA2 deleterious mutations in breast cancer in Asian countries. J Med Genet 53, 15-23.

Lanczky, A., Nagy, A., Bottai, G., Munkacsy, G., Szabo, A., Santarpia, L., et al. (2016). miRpower: a web-tool to validate survival-associated miRNAs utilizing expression data from 2178 breast cancer patients. Breast Cancer Res Treat 160, 439446.

Le, D.T., Durham, J.N., Smith, K.N., Wang, H., Bartlett, B.R., Aulakh, L.K., et al. (2017). Mismatch repair deficiency predicts response of solid tumors to PD-1 blockade. Science 357, 409-413.

Li, B., Severson, E., Pignon, J.C., Zhao, H., Li, T., Novak, J., et al. (2016). Comprehensive analyses of tumor immunity: implications for cancer immunotherapy. Genome Biol 17, 174.

Li, T., Fan, J., Wang, B., Traugh, N., Chen, Q., Liu, J.S., et al. (2017). TIMER: a web server for comprehensive analysis of tumor-infiltrating immune cells. Cancer Res 77, e108-e110.

Lian, Q., Wang, S., Zhang, G., Wang, D., Luo, G., Tang, J., et al. (2018). HCCDB: a database of hepatocellular carcinoma expression atlas. Genom Proteom Bioinform 16, 269-275.

Liu, J., Lichtenberg, T., Hoadley, K.A., Poisson, L.M., Lazar, A.J., Cherniack, A.D., et al. (2018). An integrated TCGA pan-cancer clinical data resource to drive high-quality survival outcome analytics. Cell 173, 400.e11-416.e11.

Mei, J., Hao, L., Liu, X., Sun, G., Xu, R., Wang, H., et al. (2019). Comprehensive analysis of peroxiredoxins expression profiles and prognostic values in breast cancer. Biomark Res 7, 16.

Modhukur, V., Iljasenko, T., Metsalu, T., Lokk, K., LaiskPodar, T., and Vilo, J. (2018). MethSurv: a web tool to perform multivariable survival analysis using DNA methylation data. Epigenomics 10, 277-288.

Romagnolo, A.P., Romagnolo, D.F., and Selmin, O.I. (2015). BRCA1 as target for breast cancer prevention and therapy. Anticancer Agents Med Chem 15, 4-14.

Savage, K.I., and Harkin, D.P. (2015). BRCA1, a 'complex' protein involved in the maintenance of genomic stability. FEBS J 282, 630-646.

Shan, J., Dsouza, S.P., Bakhru, S., Al-Azwani, E.K., Ascierto, M.L., Sastry, K.S., et al. (2013). TNRC9 downregulates BRCA1 expression and promotes breast cancer aggressiveness. Cancer Res 73, 2840-2849.

Shilpa, V., Bhagat, R., Premalata, C.S., Pallavi, V.R., Ramesh, G., and Krishnamoorthy, L. (2014). BRCA1 promoter hypermethylation and protein expression in ovarian carcinoma-an Indian study. Tumour Biol 35, 4277-4284.

Siegel, R.L., Miller, K.D., and Jemal, A. (2020). Cancer statistics, 2020. CA Cancer J Clin 70, 7-30.

Sun, C.C., Li, S.J., Hu, W., Zhang, J., Zhou, Q., Liu, C., et al. (2019). Comprehensive analysis of the expression and prognosis for E2Fs in human breast cancer. Mol Ther 27, 11531165.

Tai, C.S., Lin, Y.R., Teng, T.H., Lin, P.Y., Tu, S.J., Chou, C.H., et al. (2017). Haptoglobin expression correlates with tumor differentiation and five-year overall survival rate in hepatocellular carcinoma. PLoS One 12, e0171269.

Taron, M., Rosell, R., Felip, E., Mendez, P., Souglakos, J., Ronco, M.S., et al. (2004). BRCA1 mRNA expression levels as an indicator of chemoresistance in lung cancer. Hum Mol Genet 13, 2443-2449.
Thomas, L.E., Winston, J., Rad, E., Mort, M., Dodd, K.M., Tee, A.R., et al. (2015). Evaluation of copy number variation and gene expression in neurofibromatosis type-1-associated malignant peripheral nerve sheath tumours. Hum Genom 9, 3.

Tsibulak, I., Wieser, V., Degasper, C., Shivalingaiah, G., Wenzel, S., Sprung, S., et al. (2018). BRCA1 and BRCA2 mRNA-expression prove to be of clinical impact in ovarian cancer. Br J Cancer 119, 683-692.

Uhlen, M., Fagerberg, L., Hallstrom, B.M., Lindskog, C., Oksvold, P., Mardinoglu, A., et al. (2015). Proteomics. Tissuebased map of the human proteome. Science 347, 1260419.

Uhlen, M., Oksvold, P., Fagerberg, L., Lundberg, E., Jonasson, K., Forsberg, M., et al. (2010). Towards a knowledge-based Human Protein Atlas. Nat Biotechnol 28, 1248-1250.

Wang, G.H., Zhao, C.M., Huang, Y., Wang, W., Zhang, S., and Wang, X. (2018). BRCA1 and BRCA2 expression patterns and prognostic significance in digestive system cancers. Hum Pathol 71, 135-144.

Wen, W.X., and Leong, C.O. (2019). Association of BRCA1and BRCA2-deficiency with mutation burden, expression of PD-L1/PD-1, immune infiltrates, and T cell-inflamed signature in breast cancer. PLoS One 14, e0215381.

Xu, Y., Ouyang, T., Li, J., Wang, T., Fan, Z., Fan, T., et al. (2018). Predictive value of BRCA1/2 mRNA expression for response to neoadjuvant chemotherapy in BRCA-negative breast cancers. Cancer Sci 109, 166-173.

Yuan, Q., Sun, N., Zheng, J., Wang, Y., Yan, X., Mai, W., et al. (2019). Prognostic and Immunological Role of FUN14 Domain Containing 1 in Pan-Cancer: friend or Foe? Front Oncol 9, 1502.

Zhang, W., Luo, J., Chen, F., Yang, F., Song, W., Zhu, A., et al. (2015). BRCA1 regulates PIG3-mediated apoptosis in a p53dependent manner. Oncotarget 6, 7608-7618.

Zhang, W., Luo, J., Yang, F., Wang, Y., Yin, Y., Strom, A., et al. (2016). BRCA1 inhibits AR-mediated proliferation of breast cancer cells through the activation of SIRT1. Sci Rep 6, 22034.

Zheng, H., Zhang, G., Zhang, L., Wang, Q., Li, H., Han, Y., et al. (2020). Comprehensive review of web servers and bioinformatics tools for cancer prognosis analysis. Front Oncol 10, 68.

Address correspondence to: Chaoying Liu, MD

Department of Oncology Wuxi People's Hospital Affiliated to Nanjing Medical University 299 Qing Yang Road Wuxi 214023 Jiangsu China

E-mail: liuchaoying666@163.com

Huiyu Wang, PhD, MD

Department of Oncology

Wuxi People's Hospital Affiliated to Nanjing

Medical University

299 Qing Yang Road Wuxi 214023

Jiangsu

China

E-mail: wanghuiyu2011@126.com

Received for publication April 20, 2020; received in revised form June 18, 2020; accepted July 5, 2020. 